\title{
AuTOMATED DisCOVERY OF LOGICAL FALLACIES IN LEGAL ARGUMENTATION
}

\author{
Callistus Ireneous Nakpih ${ }^{1}$ and Simone Santini ${ }^{2}$ \\ ${ }^{1}$ Mathematics and Computing Department, St. John Bosco’s College of Education, \\ Navrongo, Ghana \\ ${ }^{2}$ Computer Science Department, Universidad Autonoma De Madrid, Spain
}

\begin{abstract}
This paper presents a model of an algorithmic framework and a system for the discovery of non sequitur fallacies in legal argumentation. The model functions on formalised legal text implemented in Prolog. Different parts of the formalised legal text for legal decision-making processes such as, claim of a plaintiff, the piece of law applied to the case, and the decision of judge, will be assessed by the algorithm, for detecting fallacies in an argument. We provide a mechanism designed to assess the coherence of every premise of a claim, their logic structure and legal consistency, with their corresponding piece of law at each stage of the argumentation. The modelled system checks for validity and soundness of a claim, as well as sufficiency and necessity of the premise of arguments. We assert that, dealing with the challenges of validity, soundness, sufficiency and necessity resolves fallacies in argumentation.
\end{abstract}

\section{KEYWORDS}

Artificial Intelligence, Natural Language Processing, Logic Fallacy, Legal Argumentation, Algorithms in Prolog.

\section{INTRODUCTION}

Researchers from different disciplines in Computer Science, especially Natural Language Processing (NLP), have attempted to fit different theories of human reasoning into machine computations, for resolving some important semantic challenges. Various computational tools have been developed through NLP, a discipline that is focused on the translation of human languages into formalised machine language [1][2]. Some of the NLP tools are developed to synthesise reasoning and logics of human expressions, which target different semantic challenges or errors generated in discourse [3][4].

One of the important errors generated from human discourse is fallacies. Even though fallacies form part of the natural way humans communicate, it is essentially the error of it. Fallacies generally are presented as logical faults of statements, or faulty reasoning, or both, in an argument [5][6]. Aristotle was one of the earliest philosophers who started the discussion of fallacies in a more profound way[7]. Several other classical as well as contemporary discussions have helped us to understand the nature, and to some extent the variance of expression of human thoughts that generates different types of fallacies [8][9][10]. 
However, It is still apparent that, the more the semantic gaps in natural language appears to be bridged, the more generatively complex the errors in natural reasoning and expression of language become even outward. There has been various forms of critiques [11][12] and counter critiques [13] on how fallacies have been expressed, understood, classified and applied by various researchers; it becomes quite revealing and yet interesting, that the form of fallacy itself is not simply straightforward for experts to clearly classify with compelling consistency as to what constitutes a fallacy as well as its types, and this makes it challenging for Computer Scientists to design computational systems that have the capacity to capture the complete behaviour of fallacies as a structured formalism.

Even though philosophers and argumentation theorists have provided some manual varied forms of identifying fallacies, generally in natural language and in legal language, which partly depends on sound reasoning [14], the process sometimes becomes puzzling and deserves more robust computational approaches.

\section{RELATED WORKS}

The application of NLP tools has offered diverse solutions for different challenges in legal practice. The efficiency of legal practice is largely hinged on the understanding and appropriate use of legal language; a practice that requires legal research, the access to information and the use of knowledge for legal decision-making. The Information and knowledge that governs legal practice are usually composed into statutes, regulations, procedures and so forth, which can be on different themes, but sometimes converge on account of their application on some specific issues. The understanding and use (especially in divergent legal subject issues) of legal knowledge can sometime be very tricky for the practitioners. These important challenges have attracted technologies that focus on the efficient access, understanding and use of legal information for decision-making, while NLP tools specifically target the textual structure, knowledge extraction, semantics and retrieval systems for legal language.

Callistus Ireneous Nakpih in his work "Citizenship Act (591) of Ghana as a logic Theorem and Its Semantic Implications" [15] presents legal text in a formalism that allows for computational reasoning and deduction of meaning from the text. The theorems developed guides, or rather compels a pattern of reasoning through the legal text to conform to domain specific decisionmaking processes. We recognise in that research that, ambiguities in the linguistic structure of the legal text is reduced and therefore offers a good knowledgebase system for the analysis and discovery of fallacies in legal systems. Following this idea, we provide in our model, a repository that will contain such formalism of knowledgebase of legal text that can be made accessible to a fallacy analysing mechanism.

Hiroaki Yamada, Simone Teufel and Takenobu Tokunaga developed an annotated model with algorithms for automatic extraction of argument structure based on Supervised Learning [16]. Their research work offers mechanisms that provide summaries or extracts from legal documents on individual issue topics. They evaluated the need for legal practitioners to consistently follow principles of legal arguments, an activity which can however be cumbersome in the midst of the realities of information overload for practitioners. They provided a mechanism that makes readily available, the right piece of information for a particular legal application. Our research highlights the importance for the coherence of the use and application of legal information in decisionmaking, and thus considers several indications and concepts provided in their work. Our model in this paper requires the evaluation of a piece of law employed for decision-making, to ascertain whether it forms part of a legal premise or otherwise. The concept of having access and using the 
right legal information or knowledge, as forming part of the process of efficient legal decisionmaking is thus implemented for the analysis of fallacies in our research.

There are other several impressive systems that have been modelled for decision-making, using different kinds of theories as well as drawing from other technologies which brings about improvement in the automated world. One of such impressive development is Detore and Suever's "Automated Decision-Making Arrangement" [17]. Their system executes several levels of decision operations, by receiving input data which is analysed by comparing with knowledgebase data in the system. Data that matches the system's knowledgebase is used to execute a decision operation, whereas data that do not match the knowledgebase is analysed for degree of divergence, and a decision operation is executed based on the nature of the input data. The system is generally composed of input, output, processing and storage functions. We take inspiration from the composition and function of this system, which led us to model our system to receive formalised text as input which will be transmitted into an analyser, we hold in a repository, a knowledgebase data of existing law or regulations, which will be applied to the input data in the analyser in different ways at different levels for fallacy discovery. We provide a pattern that will indicate the state of an argument being analysed in terms of soundness, validity and sufficiency.

Computational mechanisms targeting the processing of natural language for knowledge and decision-making is becoming even more impressive by the years. The IBM's Project Debater is one of the state of the art inventions that unveils a very practical feel of how machines can be made to process discourse as humans do. From this invention, we see the first Artificial Intelligent (AI) machine debate on complex topics without prior training on the topics. It has the capacity to listen to its opponent debater and give a rebuttal. The system combines complex mechanisms such as text segmentation, modelling human dilemma, listening comprehension, data-driven speech writing and delivery among others. The results of Project Debater is quite remarkable, just like several other systems that have yielded practical usefulness in various ways for resolving natural language problems.

While AI is growing and gaining the capacity to resolve various complex human problems, especially in NLP, we are yet to see robust computational mechanisms that are engineered to directly target the discovery of fallacies in natural language expressions. We therefore in this research, focus narrowly on the challenges of identifying fallacies in legal argumentation, drawing from the existing reasoning theories and mechanisms.

\section{Fallacies in Legal Argumentation}

In legal argumentation, legal practitioners try to assess the validity and soundness of the reasoning employed in the argumentation with the facts of a piece of law [18][19]. This exercise can sometimes be difficult, as legal language expression itself can be very confusing [20]. When there is a fault in the logic structure or reasoning pattern of a legal argumentation, fallacies may occur. Argumentation theorists have tried several means to classify these fallacies to capture their varied structure and complexities for identification. By these classifications, there are some form of fallacies which are being referred to as logical fallacies, where the logic rules are particularly said to be ignored in the course of making deductive conclusions [21].

Legal argumentation has been one domain of life that depends much on the use and interpretation of text for processing decisions. The interpretation of text is however subject to the complexity of legal expressions and reasoning, whereas legal expressions are mostly done with different levels 
of ambiguities which sometimes lead to unsound reasoning [22][23], and may as well result in the introduction of fallacies in decisions that are taken through the process [24].

The discovery of fallacies in the course of legal discourse or argumentation is a difficult task, and mostly eluding. This is because, the analysis of logical implication of legal expressions at the level of discourse is not robust enough to reveal faulty reasoning that lead to fallacies. Even though some researchers have attempted to use some computational means to support legal processes, they have not directly dealt with the problem of fallacies in legal argumentation to the best of our knowledge.

There are over hundred types of fallacies that have been classified by their nature and how they are presented in language. We do admit that it is quite challenging to have one computational system that can adequately process all the types of fallacies; we therefore only focus on logical fallacies that occur in legal language and have used some of the basic rules for defining non sequitur fallacies, which is a type of logical fallacy for our model.

\subsection{Non Sequitur Fallacies and Legal Argumentation}

Non sequitur fallacies are generally discussed as fallacies that have their conclusions not following their premise, however, this opinion has been disagreed by other researchers who opine that the use of non sequitur in relation to fallacies is more complex and spans beyond the definition ascribed to it [25]. In a broader sense, non sequitur fallacies occur when the logical structure of an argumentation is undermined; when the validity, soundness and sufficiency of statements are faulty [26][27] [28].

Example 1;

$$
\begin{aligned}
& \text { All birds can fly } \\
& \text { A penguin is a bird } \\
& \text { Therefor a penguin can fly }
\end{aligned}
$$

Example 2:

$$
\begin{aligned}
& \text { If } 2020 \text { is divisible by } 5 \\
& \text { then } 2020 \text { is a leap year }
\end{aligned}
$$

Example 3:

$$
\begin{aligned}
& \text { A leap year is exactly divisible by } 4 \\
& 1900 \text { is divisible } 4 \\
& \text { Therefore } 1900 \text { is a leap year }
\end{aligned}
$$

Example 1 appears to be a valid argument, that is, if in our real world it is really the case that all birds can fly, then the conclusion would be correct. This is because the conclusion relates directly to the two premise, so this is not an error of validity of the argument. However, we do recognise two dissimilar premise which are synthesised but weakens the continuity of reasoning of the argument, resulting into a false conclusion [29]. In our real world, not all birds can fly, and one of such birds is a penguin; so in the assertion, the first premise which we know is factually false is

related to a factually true premise in the statement for the deduction of our conclusion. Even though there is a semantic connection between them for the deduction process, the wrong assumption or premise of all birds can fly, poses an error of sound reasoning, since in real life not all birds can fly, which unfortunately leads to the logically correct deduction of a penguin can fly. The deductive structure followed a false premise and hence resulted into a fallacious conclusion. 
In Example 2, we do realise that the year 2020 is indeed divisible by 5 and satisfies the condition of the premise, and therefore superficially makes the conclusion true, because 2020 is a leap year. However, 2020 being a leap year has nothing to do with it being divisible by 5 . In other words, 2020 being divisible by 5 is not a criteria for determining whether it is a leap year or not, so the premise is irrelevant to the argument; even though the premise and the derived conclusion both happen to be true, the premise does not form part of the deduction and is not necessary for the conclusion.

In Example 3, we also realise that, we have two true premise for deducing the conclusion; it is true that a leap year is divisible by 4 , and it is also true that 1900 is divisible by 4 , however, the conclusion that 1900 is a leap year is false. This is because, the premise given in the argument are not sufficient to deduce the conclusion even though they are true, there are other factors which are needed in addition to the stated premise to correctly arrive at the conclusion that a year is a leap year or otherwise. These examples typifies the general form and structure of logical fallacies, which are generated by the different types of errors as shown.

Non sequitur fallacies are common in legal argumentation, in the sense that, some claims made usually do not have sufficient or relevant premise to justify alleged conclusions made by the claimer, or, the reasoning employed to state an allegation is either faulty or not valid; the laws of the court requires that, any person making an allegation against an opponent has the onus to tender in evidence of their claim to the court for examination and decision-making.

The exercise of assessing and deciding whether a claim is true or false typically follows the pattern of asserting a non sequitur fallacy in legal argumentation; in both instances of the processes, we are generally interested in whether the conclusion made follows its corresponding premise; this close relation of the processes allows us to narrow our solution to identify non sequitur fallacies in legal argumentation which are logical and deductive errors in nature, by establishing the disconnect between a premise and a conclusion. We are also interested in ensuring that, claims made are done with legal certainty [30].

\section{FINDINGS}

In this study, we generally provide a modelled system that examines all aspects of a legal statement using rules that establish validity, soundness, sufficiency and necessity, for the automated discovery of fallacies; we assert from the finding of this study, that, an argument that is valid, sound and has sufficient and necessary propositions avoids fallacies. The computational mechanism provided by this study therefore requires that an argument is assessed in all these aspects.

Logical fallacies occur in the process of reasoning where the logics of an argument is undermined. We have only maintained the generic structure of Non Sequitur fallacies for their deductions in legal text, and not really considering all specific forms and complexities of it. The

generic structure maintained here is sufficient for the purpose and delimitation of this study. Non sequitur fallacies in legal argumentation can occur generally in the following;

1. A claim made against an opponent or a respondent.

2. Facts presented to a court by a respondent against a claim. 
3. The law or legislative instrument employed for judgement or decisionmaking of the court.

4. The conclusion or decision of a judge on a case.

The modelled system considers the enlisted areas above for assessment and the discovery of fallacies. Usually, in a court case, a claim follows a logic structure of antecedent and consequent to challenge real facts of a respondent based on a legal backing [31]. In our model, a claim $\mathrm{Cl}$ has a set of premise $P c$, where $P c$ holds a list of statements $P c_{1}, P c_{2}, \ldots, P c_{n}$. and $C l$ is implied by $P c$.

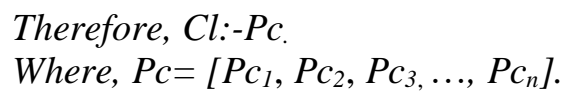

Claims are truth-functional which are usually asserted using corresponding or appropriate laws. We denote an existing piece of law that would be employed to assert a claim as $L w$. The law is also an argument composed with antecedent-consequent logic structure, and sometimes as axiomatic facts. We denote the premise of a piece of law as $P L$, with atomic statements $P L_{l}$, $P L_{2} \ldots P L_{n}$.

$$
\begin{aligned}
& \text { Therefore, } L w:-P L \text {. } \\
& \text { Where, } P L=\left[P L_{1}, P L_{2}, \ldots, P L_{n}\right] \text {. }
\end{aligned}
$$

We denote the decision of the judge or the court as $D$ with a list of premise $P D$, with atomic statements $P D_{1}, P D_{2}, \ldots, P D_{n}$. Which are based on legal provision.

$$
\begin{aligned}
& \text { Therefore, } D:-P D . \\
& \text { Where, } P D=\left[P D_{1}, P D_{2}, P D_{3}, \ldots, P D_{n}\right] \text {. }
\end{aligned}
$$

The defined premise and implied conclusions are variables that hold atomic statements defined in the law and the claim, which are all truth-functional. Generally, the mechanism for assessing legal and logic requirements of an argument is provided for in the model.

\subsection{Algorithmic Model for Automated Fallacy Discovery}

The algorithm is designed to assess any claim, as well as associated legislative instrument employed by a court. It also uses facts established by the court and performs a deductive reasoning through the text for the discovery of logical fallacies that may occur in the process.

\section{The Algorithm}

Fetch $\mathrm{Cl}$.

Fetch Lw.

$D O$ Validity $(\mathrm{Cl})$.

If FALSE PRINT "Fallacy Alert - invalid Claim” else GOTO NEXT

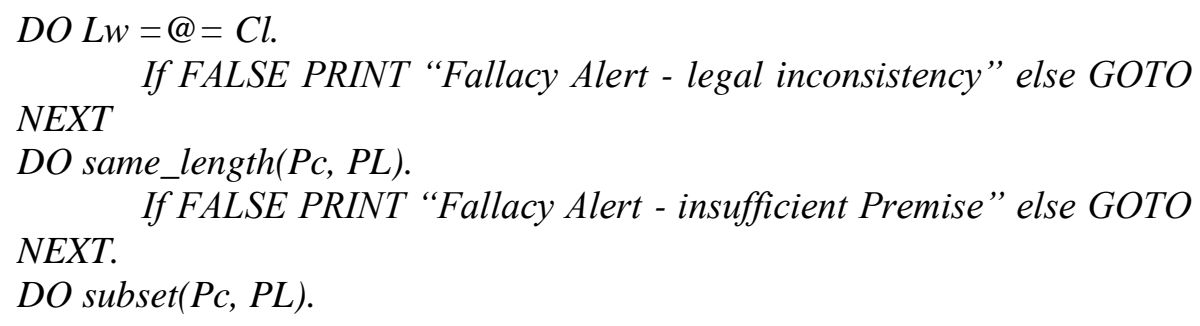


International Journal of Artificial Intelligence and Applications (IJAIA), Vol.11, No.2, March 2020

DO ?-CL.

If FALSE PRINT “Fallacy Alert-Not Necessary Premise” else

STOP.

\section{Prolog code for establishing a fallacy;}

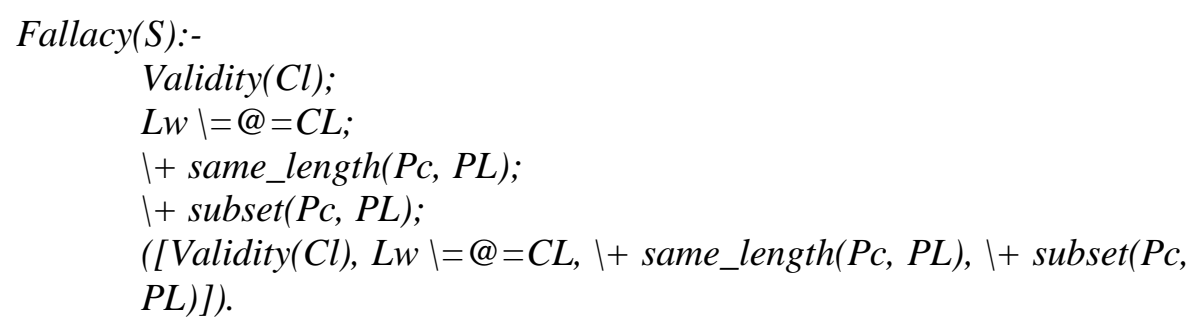

We propose for our system, an input mechanism that can transmit a claim generated in Prolog to a fallacy analyser, that will receive the claim and as well receive the corresponding piece of law in Prolog programme from a Knowledgebase. The analyser will use the pattern provided in the algorithm to assess the validity, soundness, sufficiency and necessity as well as the legal backing of an argument. The analyser after these assessments can then process facts of a respondent, against whom a claim is made to ascertain the truth values of the whole argument.

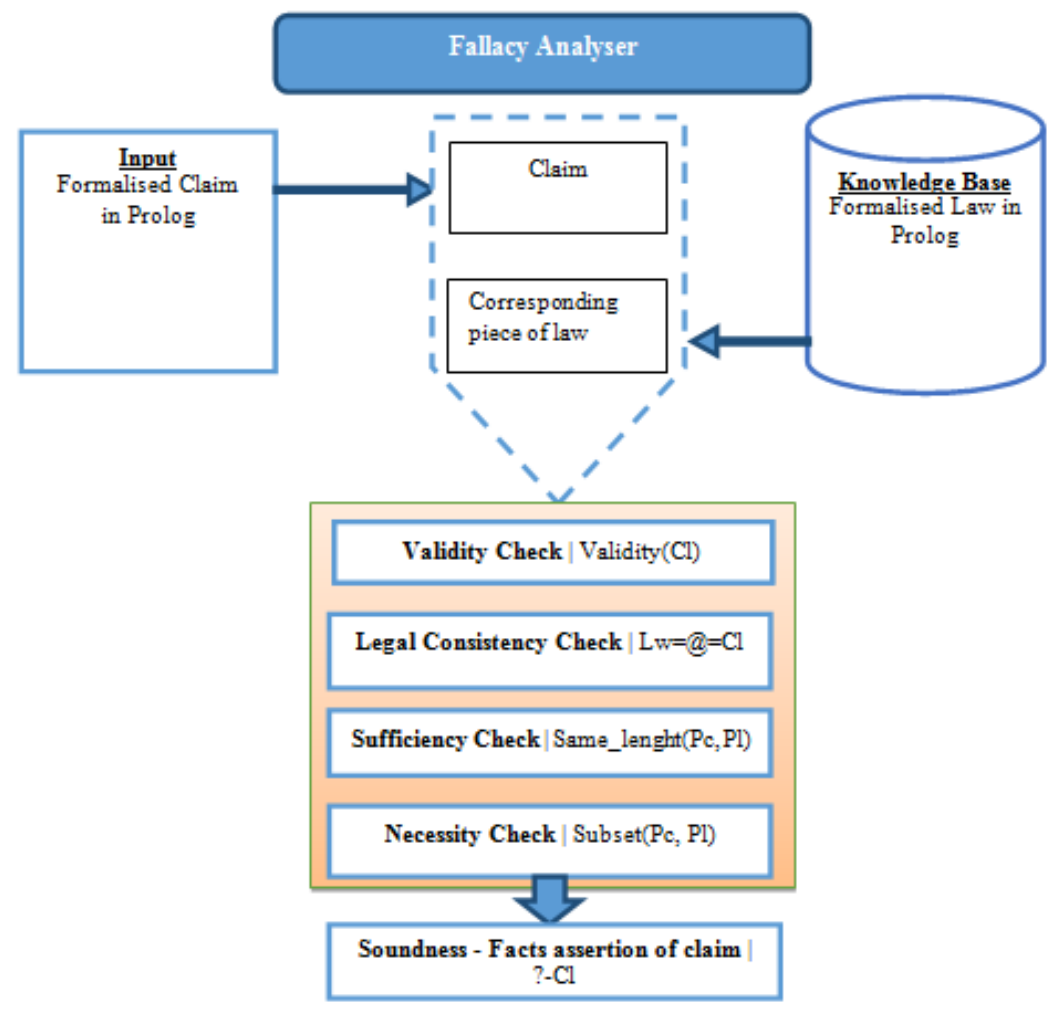

Figure 1. Nakpih’s Model of Automated Fallacy Discovery System 


\subsection{Validity and Soundness}

Some researchers argue that validity of logics, especially of the First Order, is undecidable and also semi-decidable [32][33]; however, the general rules for establishing validity suffices for the process in our model, that is, an argument is said to be valid if and only if the conclusion cannot be false whiles all premise of the argument are true [34], which can be proven through various means including contrapositive [35][36]. The model below ensures validity of a claim;

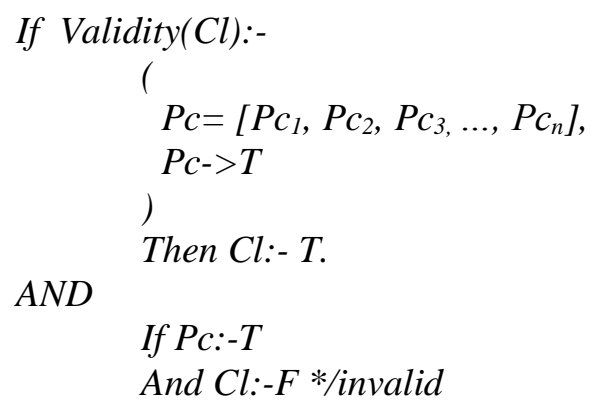

For a claim to be in a valid logic form, all atomic statements of the premise $P c_{1}$ to $P c_{n}$ if $A N D e d$ together evaluates a True value $(T)$, then the conclusion of the claim must resolve True. However, if the ANDed atomic values of $P c$ resolves True and the conclusion of the claim resolves False $(F)$, then the argument or claim is invalid.

One other step in the decision-making process on a court case, is to examine evidence of claims against the real facts of a respondent [37]. This is where the truth-functionality of propositions is tested. The model therefore allows for the evaluation of truth values of propositions of claims against the certified real facts of a respondent, and thus maintains the soundness of an argument.

A sound argument must first be valid and must have premise which are really true [38]. While validity maintains that the structural composition of a claim guarantees that a conclusion should be true as long as its premise are true, the soundness of a claim further enforces that the given premise are actually true [39]. The component of the model enforcing soundness also evaluates the content of a claim against established facts of the court to ascertain truth values of the claim (after its logic form is satisfied). This will allow for evidence or facts of a claim to be evaluated through deductive reasoning; the important facts established by court will be given as input to the system for the evaluation process. The objective here is to ensure that, claims are not only valid but sound, and this is because, sound arguments to a good extend resolves fallacies.

\subsection{Sufficiency and Necessity}

On the account of sufficiency for the avoidance of fallacy [40], we ensure that the minimal premise required to establish a conclusion of an argument is enforced. By this evaluation, claims made without adequate required premise to deduce a conclusion will be detected. The sufficiency of the premise of a claim is evaluated based on legal provision; every piece of law usually has a set of premise, or, axiomatic facts that are used to establish legal conclusions. The premise of a claim therefore should not be anything less than what is required by a corresponding piece of law for the deductive process. Thus, the obvious minimal premise for a legal conclusion must always be maintained. We therefor generate same_length $(P c, P L)$ where the premise of the claim is matched with the required premise of piece of law, for ensuring sufficiency of an argumentation. 
On the account of necessity [41], evaluation of a claim and its associated law is also made; the evaluation is done to ascertain whether the claim is made on valid legal grounds or otherwise, even before the process of fact-finding is engaged. Claims generated must correspond to some legal provision to allow for legal reasoning. The atomic statements made in a claim should have some coherence with atomic statements made in the declaration of its associated law; we generate $L w=@=C l$ to assess the structural equivalence of a piece of law corresponded to a claim. We as well generate $\operatorname{subset}(P c, P L)$ to ensure that the premise of a claim relates to the premise of an existing law. This is to warrant that the premise of a claim made is a legal premise and is relevant or necessary for the reasoning and deduction of a legal consequent in a claim. By this evaluation, a premise which is found in a claim but not relevant or necessary for the deduction process per legal provision will be detected.

The various aspects of the model ensures that different logical and legal requirements of an argument are asserted, and their combined process guarantees the detection of fallacies.

\section{FUTURE WORK}

The system we have provided analyses formalised text for fallacies, however, the processes of formalism of natural text is not automatic. For future works and for the improvement of our system, it will be interesting to provide a mechanism that can extract natural text and formalise it automatically into a knowledgebase where it can be further be retrieved for the fallacy analysis.

We also look forward to a mechanism that can automatically retrieve a piece of law related to a claim. The ability to identify a piece of law that is appropriate for assessing the legal consistency of a claim is a human activity in our system. An intelligent mechanism for retrieving a piece of law automatically based on a claim will augment the efficiency of our model. There are several information retrieval systems for legal practice, which generally retrieve documents required with content for use, such retrieval mechanisms can be modelled to focus on understanding formalised claims and then retrieve the corresponding legislative instrument from a knowledgebase for fallacy analysis. Formalism of fallacies is a grey area in NLP, other researchers can take the leverage of using theories and technologies that exist already to target this complex problem of natural language.

\section{CONCLuSion}

The quest to resolve fallacies in argumentation is not a simple task; fallacies have been controversial since the discussions of it begun, and still remains controversial, so, we do not claim to offer a computational solution that captures all the complexities of logical fallacies. Meanwhile, various research discussions have given credible insight to the general structure and form of fallacies. The main concern for language users in general, especially legal practitioners, is how to be able to identify these fallacies when they occur, or rather, how to avoid them all together. Through NLP, which is a branch of AI, researchers have provided various tools for addressing different semantic challenges; we therefore follow the insight on fallacies and the technologies of NLP available to us for providing a system that discovers logical fallacies in legal argumentation automatically.

In this paper we provided a computational mechanism for assessing legal argumentations, for the discovery of logical fallacies, particularly non sequitur; we consider the problem of validity, soundness, sufficiency and necessity among other factors as underpinning the generation of logical fallacies in legal argumentation, we therefore provided a model that allows these aspects of any legal claim or argument made to be evaluated. At each step of evaluation by the modelled 
system, we ensure that any error realised in a statement that amounts to a non sequitur fallacy can be detected. We generated an algorithm in Prolog, and then a conceptual model of how the whole system is intended to work for the discovery of fallacy. We thus assert by this research, that dealing with the problem of validity, soundness, sufficiency and necessity resolves fallacies in argumentation.

\section{ACKNOWLEDGEMENTS}

We acknowledge our friends in the legal fraternity who offered us very insightful guidance for understanding legal processes and argumentation

\section{REFERENCES}

[1] T. Bell (2018) "what is Natural Language Processing? The Business benefits of NLP Explained" CIO from IDG, accessed 18th September 2019, from $<$ https://www.cio.com/article/3258837/artificialintelligence/ what-is-natural-language-processing-thebusiness-benefits-of-nlp-explained.html>

[2] U.S. Tiwary and T. Suddiqui (2008) "Natural Language Processing and Information Retrieval", Oxford University Press Inc., New York, NY USA.

[3] P. Blackburn, J. Bos (2003) "Computational Semantics", THEORIA. An international Journal for Theory, History and Foundation of Science, Vol. 18, No. 1, pp. 27-45.

[4] J.R. Smith (2007) "The Real Problem of Bridging the 'Semantic Gap' ". In: Sebe N., Liu Y., Zhuang Y., Huang T.S. (eds) Multimedia Content Analysis and Mining. MCAM 2007. Lecture Notes in Computer Science, Vol. 4577. Springer, Berlin, Heidelberg.

[5] H.J. Gensler (2010) "The A to Z of Logics", Rowman \& Littlefield, pp. 74.

[6] R. Paul and L. Elder (2008). "The thinker's guide to fallacies: The art of mental trickery and manipulation." Dillon Beach, CA: Foundation for Critical Thinking Press.

[7] Aristotle (1955) “On Sophistical Refutations” Loch Classical Library, Cambridge, Mass., Harvard

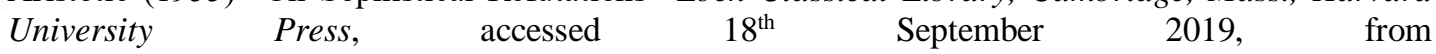
<http://www.hup.harvard.edu/catalog.php?isbn=9780674994416>

[8] A. Pineau (2013) “Informal Logic" Vol. 33, No. 4, pp. 531-546, McMaster University, Canada.

[9] R.H Johnson and J.A. Blair (1993). "Dissent in fallacyland, Part 2: Problems with Willard". In R.E. McKerrow (Ed.), Argument and the postmodern challenge: Proceedings of the eighth NCA/AFA conference on argumentation, pp. 191-193. Annandale, VA.

[10] R.H. Johnson, and J.A. Blair (1983) "Logical Self-Defense", Second Edition, McGraw Hill Ryerson, Toronto.

[11] C.L. Hamblin (1970) "Fallacies", London: Methuen.

[12] R.H. Johnson (1990) "Acceptance is not Enough: A Critique of Hamblin", Philosophy \& Rhetoric, Penn State University Press, Vol. 23, No. 4, pp. 271-287.

[13] D.N. Walton (1991) "Hamblin on the Standard Treatment of Fallacies", Philosophy \& Rhetoric, Penn State University Press, Vol. 24, No. 4. pp. 353-361. 
International Journal of Artificial Intelligence and Applications (IJAIA), Vol.11, No.2, March 2020

[14] H. Hans (2019), "Fallacies", The Standard Encyclopaedia of Philosophy, Metaphysics Research Lab, Standford University.

[15] N. I. Callistus (2018) "Citizenship Act (591) of Ghana As A Logic Theorem And Its Semantic Implications”, International Journal on Natural Language Computing, Vol. 7, No. 5, pp. 11-26.

[16] H. Yamada, S. Teufel and T. Tokunaga (2017), "Designing an annotation scheme for summarizing Japanese judgment documents," 9th International Conference on Knowledge and Systems Engineering (KSE), Hue, pp. 275-280.

[17] W. A. Detore and R.D. Suever (1998) "Automated Decision-Making Arrangment", United States National Risk Management, Inc. (Fort Wayne, IN), Patent: 5732397.

[18] N. Luhmann (1995) "Legal Argumentation: An Analysis of Its Form”, Modern Law Review, Vol. 58, No. 3. pp. 285-298.

[19] J. Dickson (2010) "Interpretation and Coherence in Legal Reasoning", The Stanford Encyclopedia of Philosophy, Metaphysics Research Lab, Standford University.

[20] V. Jacqueline (2009), "Speech acts in Legal Language: Introduction", Journal of Pragmatics $-J$ PRAGMATICS, Vol. 41, pp. 393-400.

[21] J.W. Stanley (1872) "Elementary Lessons in Logic: Deductive and Inductive", MacMillian and Co., London.

[22] A. Wagner and S. Cacciaguidi-Fahy (2006) "Legal Language and the search for Clarity: Practice and tools", Peter Langi.

[23] H. Singh (2013) "Language of Law - Ambiguities and Interpretation", International Association of Scientific Innovation and Research, USA, pp. 122-126.

[24] B. G. Slocum (2010) “The Importance of Being Ambiguous: Substantive Canons, Stare Decisis, and the Central Role of Ambiguity Determinations in the Administrative State" Maryland Law Review, Vol. 69, No. 4.

[25] J. Corcoran (2015) “Meaning of Non Sequitur”, State University of New York, Buffalo.

[26] J.A. Blair (1995), "The place of teaching informal fallacies in teaching reasoning skills or critical thinking," In Hansen and Pinto 1995, pp. 328-38.

[27] D.N Walton (2011) “Defeasible reasoning and informal fallacies” Synthese, Vol. 179, pp. 377-407.

[28] F. Rosen (2006) "The philosophy of error and liberty of thought: J. S. Mill on logical fallacies," Informal Logic, Vol. 26, pp.121-47.

[29] Mark van Atten and Dirk van Dalen (2002) “Arguments for Continuity Principle”, Bulleting of Symbolic Logic. 8, pp. 329-347.

[30] A. Alexy (2015) "Legal Certainty and Correctness", International Journal of Jurisprudence and Philosophy of Law, Vol. 28, No. 4, pp. 441-541.

[31] Justice (2017) "How to Start Proceedings-The Claim Form", Ministry of Justice, UK, accessed 22 nd August 2019, from < https://www.justice.gov.uk/courts/procedure-rules/civil/rules/part07>

[32] J. Worrell (2016) “Undecidability of Validity and Satisfiability”, Logic and Proof, Hilary. 
International Journal of Artificial Intelligence and Applications (IJAIA), Vol.11, No.2, March 2020

[33] J. Hoenike (2012) "Validity of FOL is Undecidable", accessed $21^{\text {st }}$ November 2019, from $<$ https://swt.informatik.unifreiburg.de/teaching/SS2012/DecisionProcedures/resources/folIsUndecidab le.pdf>

[34] Internet Encyclopedia of Philosophy (IEP) "Validity and Soundness", accessed 21 st November 2019, from < https://www.iep.utm.edu/val-snd/>

[35] T. Peil (2006) "Logic Argument and Constructing Proofs", Minnesota State University, access $21^{\text {st }}$ November 2019, from < http://web.mnstate.edu/peil/geometry/Logic/6logic.htm>

[36] G. Kemerling (2011) "Proof-Procedures for Propositional Logic", Philosophy Pages, accessed 21 ${ }^{\text {st }}$ November 2019, from < http://www.philosophypages.com/lg/e11c.htm>

[37] R.S. Summers (1999) "Formal Legal Truth and Substantive Truth in judicial Fact-Finding: Their Justified Divergence in Some Particular Cases", Law and Philosophy, Springer, Vol. 18, No. 5, pp. 497-511.

[38] P. Girard "Sound and Cogent Argument", Logic and Critical Thinking, University of Aukland, accessed $13^{\text {th }}$ November 2019, from https://www.futurelearn.com/courses/logical-and-criticalthinking/0/steps/9152

[39] J.Y.F. Lau (2011), “An Introduction to Critical Thinking and Creativity: Think more, Think better", Hoboken, N.J. Wiley.

[40] M. Walker (2011) "Critical Thinking by Example", accessed 21 $1^{\text {st }}$ November 2019, from < http://www.criticalthinkingbyexample.com/Thecompletebook/1.1\%20CTBE\%20Book\%20June\%202 011.pdf >

[41] N. Swartz (1997) "The Concept of Necessary Conditions and Sufficient Conditions", Simon Fraser University, Department of Philosophy, accessed 20 $0^{\text {th }}$ November 2019, from <https://www.sfu.ca/ swartz/conditions1.htm> 